\title{
Les paradoxes de la résilience en matière de sécurité alimentaire ${ }^{1}$
}

\author{
Abigaiil Fallot \\ François Bousquet
}

Sandrine Dury

Mots-clés

développement agricole, trajectoires, seuils, interactions, irréversibilité, transdisciplinarité, approche, participation

Résumé

L'article analyse les correspondances et les décalages entre la pensée sur la résilience dans la littérature et la façon dont on traite de résilience en matière de développement et de sécurité alimentaire dans les projets et dans les guides pratiques d'évaluation. Six paradoxes sont soulevés à propos : de la compréhension ou de la mesure de la résilience; de la résilience en situation de blocage ; du rôle de métaphore mobilisatrice du concept alors que son interprétation prête à controverses; de son caractère intégrateur alors que son évaluation porte, le plus souvent, sur des composantes dissociées; de son positionnement par rapport à la vulnérabilité ; et de la pluridisciplinarité propre au concept de résilience. On cherche comment résoudre chaque paradoxe et mieux saisir les liens entre production agricole et sécurité alimentaire des ménages ruraux dans des contextes de pauvreté, de dégradation environnementale et d'aléas multiples. On souligne ainsi ce qui singularise les travaux sur la résilience en termes d'approche et la possibilité d'une contribution originale dans le domaine du développement, avec notamment la prise en compte des points de vue en présence et un éclairage sur les irréversibilités des changements considérés.

\section{The Resilience Paradoxes Regarding Food Security}

Keywords

agricultural development, trajectories, thresholds, interactions, irreversibility, transdisciplinary approach, participation

Abstract

\footnotetext{
${ }^{1}$ La réflexion sur la résilience, dont est issu cet article, a été menée dans le cadre du projet RELAX $\left(\mathrm{N}^{\circ} \mathrm{AF} 1507-\right.$ 329 ; $\mathrm{N}^{\circ}$ FC 2015-2440, $\mathrm{N}^{\circ}$ FDNC Engt 00063479), financé dans le cadre de l'initiative «Thought for Food » par Agropolis Fondation (sur programme Investissement d'avenir, financement ANR-10-LABX-0001-01), par Fondazione Cariplo et la Fondation Daniel et Nina Carasso.

Les auteurs remercient pour leurs commentaires et pour leurs suggestions d'améliorations : Emmanuelle Bouquet, plusieurs relecteurs anonymes de cet article et les éditeurs de la revue.
} 
The article analyses the conformities and mismatches between thinking about resilience in the literature and how resilience is addressed in projects for development and food security and practical guides for resilience assessment. Six paradoxes are raised, regarding: the understanding or measurement of resilience; resilience in a situation of blockage; the role of the concept as a mobilizing metaphor when its interpretation is controversial; its integrative nature when its evaluation most often focuses on dissociated components; its positioning in relation to vulnerability; and the multidisciplinary nature of the concept of resilience. We consider how to solve each paradox and better understand the links between agricultural production and rural household food security in a context of poverty, environmental degradation and multiple hazards. This highlights what can distinguish the work on resilience in terms of approach, and the possibility of an original contribution in the field of development, namely by considering the diversity of points of view involved and by shedding light on the irreversibility of changes. 


\section{Introduction}

Le concept de résilience est mobilisé par un nombre croissant de disciplines de la recherche agronomique pour le développement et d'acteurs du développement (Lallau, 2014 ; Dubois et Ouattara, 2014). Ces disciplines et ces acteurs abordent conjointement les enjeux de sécurité alimentaire et de gestion des ressources (sols, eau, biodiversité) dans des contextes de persistance de la pauvreté et de dégradations environnementales, problèmes exacerbés par la fréquence accrue d'extrêmes climatiques (Korbéogo, 2016 ; Sanou et Badolo, 2017).

L'usage du concept de résilience pour l'analyse et la décision face à des aléas s'est généralisé dans des contextes variés (Koffi, 2014 ; Mathevet et Bousquet, 2014). À l’instar du qualificatif «durable», le mot « résilience » est entré dans le vocabulaire courant, gagne les plaidoyers et devient un critère pour des interventions d'adaptation au changement climatique et de sécurité alimentaire (O’Connell et al., 2016).

La pensée sur la résilience a aussi évolué (Folke, 2016 ; Bousquet et al., 2016 ; Quinlan et al., 2016 ; Béné, 2017) avec des définitions et des méthodologies visant à préciser ce qu'est la résilience et comment l'évaluer. Dans le domaine du développement, le concept de résilience est mobilisé en lien avec les problématiques de vulnérabilité : pauvreté, adversités et crises (Adger et al., 2005; Béné et al., 2012 ; Cannon et Müller-Mahn, 2010 ; Pelling, 2011). Les organisations internationales et les organisations non gouvernementales (ONG) qui ont fait de la résilience un objectif de leurs interventions (Ancey et al., 2014) cherchent à en mesurer les effets.

Cette généralisation mène souvent à des injonctions (à la transformation) et à des vœux pieux (sur l'adaptation). Elle est propice à des malentendus dans les domaines du développement et de la gestion des ressources naturelles. Dans quelle mesure la résilience remet-elle en question le développement ? S'agit-il de prévenir contre des modes de développement qui fragilisent les populations et qui épuisent les ressources? Risque-t-on de renoncer à des objectifs de lutte contre la pauvreté et de développement au nom de la résilience, notamment dans le contexte de changements globaux qui affectent les agricultures familiales au Sud (Béné et al., 2014) ? Cela explique-t-il que, comme le soulignent Lallau, Laissus-Benoist et Mbetid-Bessane (2018), la notion de résilience est encore très peu appropriée, ou de manière simpliste, par certains acteurs : autorités locales, services nationaux étatiques, fédérations paysannes?

Dans ce contexte, on s'interroge ici sur la portée du concept de résilience en matière de développement, d'environnement et de sécurité alimentaire des ménages ruraux. Nous analysons si la façon dont on pense la résilience, dans la sphère académique ("resilience thinking", que nous traduisons par le "penser résilience »), correspond à la façon dont les projets et les guides pratiques d'évaluation s'en emparent. Ce croisement nous amène à identifier six paradoxes que nous décrivons et que nous discutons dans cet article, à partir d'une étude bibliographique.

La littérature académique sur la résilience est examinée à partir de plusieurs synthèses de la diversité des définitions et approches (Bousquet et al., 2016 ; Brand et Jax, 2007 ; Quinlan et al., 2016) et de plusieurs analyses critiques (Fisichelli et al., 2016 ; Janin et Roy, 2016 ; Lallau, 2014). On étudie les développements 
théoriques en matière de résilience et de développement (Barrett et Constas, 2014 ; Lade et al., 2017). Toutefois, si cette revue n'est pas exhaustive sur la résilience et ne fait pas l'inventaire de ses définitions, elle considère deux écoles de la résilience pour traiter des relations entre développement, environnement et sécurité alimentaire : la résilience des systèmes écologiques et sociaux (SES) et la résilience issue du développement (Botta et Bousquet, 2017). Pour Dubois et Ouattara (2014), ces deux courants s'accompagnent de celui de la résilience sociale, qui s'étudie par la capacité des acteurs sociaux à surmonter l'impact de crises ; pour d'autres, les deux écoles prennent en compte une résilience sociale en s'intéressant en particulier à la résilience des communautés (Adger, 2000). La résilience des SES prend en compte les institutions et les communautés concernées pour en déduire la trajectoire du SES, alors que la résilience dans le domaine du développement regarde comment les ménages et les individus dépendent de la résilience des communautés et y contribuent, notamment avec les ressources partagées. Quelle que soit la catégorisation adoptée, on considère ici qu'un ménage et «son » exploitation agricole (entités économiques qui ne se recouvrent pas systématiquement) forment un système. On se positionne directement dans les domaines du développement et en lien avec des approches systémiques susceptibles d'intégrer des aspects économiques, écologiques et sociaux. On s'intéresse aux travaux à l'échelle des ménages ruraux et de leurs exploitations, avec, d'une part, à plus petite échelle, les individus qui constituent ces ménages et leurs ressources, et, d'autre part, à une échelle plus large, les communautés et territoires dont font partie les ménages et leurs exploitations.

Les guides pratiques d'évaluation analysés sont ceux qui font référence dans des projets de développement et d'aide humanitaire sans être spécifiques à une problématique en particulier (par exemple, l'aide aux réfugiés ou la résilience des villes), à moins de concerner la sécurité alimentaire des ménages ruraux. Les rapports de Schipper et Langston (2015), et de la FAO (2015) répertorient une trentaine de méthodes servant à l'évaluation de la résilience.

Ces méthodes relèvent de cinq principales initiatives en matière d'évaluation, qui ont donné lieu à différents guides où les concepts de la résilience sont opérationnalisés et susceptibles de traiter d'agriculture, de gestion environnementale et de sécurité alimentaire :

- RIMA (Resilience Index Measurement and Analysis) de l'Organisation des Nations unies pour l'alimentation et l'agriculture (FAO, 2016 - 80 p.) ;

- BRACED (Building Resilience and Adaptation to Climate Extremes and Disasters) de l'aide du Royaume-Uni (BRACED Myanmar Alliance, 2016 - 76 p.) ;

- COBRA (Community Resilience Assessment And Action) du Programme des Nations unies pour le développement (UNDP, 2014 - 24 p.) ;

- RAPTA (Resilience, Adaptation Pathways and Transformation Assessment) du Groupe consultatif scientifique et technique du Fonds pour l'environnement mondial (O'Connell et al., $2016-112$ p.) ; 
- le manuel Assessing Resilience in Social-Ecological Systems de l'Alliance pour la résilience (Resilience Alliance, 2010 - 54 p.).

- On inclut aussi l'outil DReW (Dynamic Resilience Wheel) du Secours luthérien mondial (LWR, $2016-22$ p.).

Le tableau 1 résume les orientations respectives de ces méthodes et outils.

\section{Tableau 1 : Principales initiatives en matière d'évaluation de la résilience pour les projets de développement agricole et sécurité alimentaire}

Il existe un paradoxe lorsqu'on identifie une ambiguité et un possible décalage entre la pensée sur la résilience et la façon dont les projets de développement la promeuvent, la traitent et l'évaluent.

\section{Mieux on comprend, moins on mesure}

Le premier paradoxe, déjà souligné par Dubois (2016), est que mieux on comprend la résilience, moins on sait la mesurer, et plus on mesure la résilience, moins on en saisit la signification spécifique. Comprendre la résilience, c'est, en fin de compte, parvenir à la définir. Depuis plus de trente ans, les définitions de la résilience sont nombreuses et relèvent de différents points de vue (Quinlan et al., 2016). Résilience selon l'ingénieur, selon le psychologue, écologique, socio-écologique, sociale, socio-économique, résilience dans le développement, résilience des communautés, la résilience caractérise la façon de réagir à une adversité ou une perturbation (Vonthron etal., 2016). Une perturbation peut être un choc ou une crise, un événement exceptionnel ou les difficultés résultant de sa répétition, mais aussi une intervention d'aide ou de politique publique (Resilience Alliance, 2010).

Avec Holling et la résilience écologique (1973), la pensée sur la résilience a été associée à une vision systémique qui appréhende les liens entre les composantes d'un système. Et en considérant que l'humain fait partie de la nature, on s'intéresse à des socio-écosystèmes (SES) ou systèmes socio-écologiques (Berkes et Folke, 1998). Ils sont qualifiés de systèmes adaptatifs complexes, du fait qu'ils s'adaptent et s'auto-organisent en réponse à une perturbation interne ou externe, ce qui génère des dynamiques nonlinéaires, des cycles adaptatifs. La pensée sur la résilience rompt donc avec la vision linéaire, ou stop-and-go, du développement économique, qui porte essentiellement sur l'évolution de la production et des revenus dans le temps.

La résilience relève des phénomènes de différentes natures qui marquent l'évolution des composantes d'un SES et de leurs liens: processus écologiques, économiques, sociaux, etc. D’un point de vue pluridisciplinaire donc, la résilience est la capacité pour un ménage, un territoire ou autre entité faisant 
système, de faire face à des perturbations : les absorber, s'adapter ou se réorganiser (Folke et al., 2010), (voir fig. 1).

Figure 1: L'éventail de capacités auxquelles fait référence le concept de résilience

$\begin{array}{ccc} & \text { Intensity of change / transaction costs } & \\ \text { stability } & \text { flexibility } & \text { change } \\ \text { Absorptive coping } & \text { Adaptive } & \text { Transformative } \\ \text { (persistence) } & \text { Capacity } & \text { Capacity } \\ & \text { (incrementaladjustment) } & \text { (transformational responses) }\end{array}$

Source : Béné et al., 2012.

Plus largement, la résilience est la capacité à vivre avec le changement, voire la notion de le mettre à profit (Mathevet et Bousquet, 2014). Les perturbations n'étant pas toujours prévisibles et leurs conséquences dépendant de plusieurs processus reliés imparfaitement connus, la résilience suppose de traiter avec l'incertitude et la complexité (Katrina Brown, plénière de la conférence «Resilience 2017 »). Dans ce contexte, comprendre la résilience revient à saisir les mécanismes en jeu dans les réponses aux perturbations. Dans cette optique, les guides d'évaluation de la résilience expliquent comment décrire un système et ses dynamiques en interaction à partir de la formulation d'une problématique et des processus qui l'alimentent ou qui la font évoluer (BRACED Myanmar Alliance, 2016; O’Connell et al., 2016; Resilience Alliance, 2010). Mais ces guides ne précisent pas comment mesurer la résilience et sur quoi doit porter la mesure exactement. Comme l'écrivent Quinlan et al., cités dans Bousquet et al. (2016, p. 10):

De nombreux chercheurs conviennent qu'il est possible de mesurer la résilience, mais qu'il faudra aussi tenir compte du fait que la résilience est un moyen et non une fin pour trouver des indicateurs et des mesures correspondant aux caractéristiques clés du concept ${ }^{2}$.

Or, dans le domaine du développement, la résilience est souvent devenue un objectif au sein des projets, dont la réalisation doit être évaluée, notamment pour rendre des comptes aux bailleurs de fonds et pour faciliter le suivi de projets successifs. Les propositions existantes de mesure de la résilience portent généralement sur les caractéristiques de ce dont on évalue la résilience, un ménage par exemple, et les réserves ou ressources que ce dernier peut mobiliser pour faire face aux perturbations. Dans le domaine du développement et de la sécurité alimentaire (FAO, 2016 ; UNDP, 2014), la résilience se mesure à l'aune des actifs du ménage ou «moyens d'existence», de l'anglais livelihood (Scoones, 1998). Des indices de résilience sont calculés à partir de variables proxies des «piliers de la résilience » (accès aux services de base,

2 Traduction des auteurs. 
actifs, filets de sécurité, capacité adaptative) ou des «capitaux» (humain, naturel, financier, social et physique). Mesurer ainsi la résilience ne permet pas forcément de la comprendre. D’une part, l'existence de ressources pour faire face aux chocs ou difficultés ne garantit pas leur adéquate mobilisation. « Reste à définir clairement ce qui manque dans les mesures de résilience ou ce qui peut y être éludé quand on change d'objet central, de la compréhension de la résilience d'un système, à la mesure de cette dernière ${ }^{3}$ » (Bousquet et al., 2016, p. 10). D’autre part, ce type de mesure occulte la définition des éléments du système considéré et plus encore les liens entre ces éléments.

Par exemple, dans le domaine de la sécurité alimentaire, où la diversité de l'alimentation des femmes est déterminante, Lourme-Ruiz et al. (2016) montrent qu'au Burkina Faso, les revenus des ménages ruraux jouent moins sur la diversité de l'alimentation que les liens intra-ménages définissant les transferts et réajustant l'argent dont disposent les femmes. Au Burkina Faso toujours, Kevane et Gray (1999) soulignent la complexité et la forte variabilité dans le temps des droits fonciers selon lesquels les femmes sont impliquées dans la production agricole. Dans ce contexte, une simple photographie des droits d'accès, à un instant donné, ne permet pas réellement de statuer sur le niveau d'empowerment féminin ${ }^{4}$, la situation alimentaire et la résilience des femmes et de leur famille.

Résoudre le paradoxe de la compréhension versus la mesure de la résilience suppose de considérer conjointement les ressources et les mécanismes de la résilience : les actifs et capacités des ménages, ainsi que les processus selon lesquels ces composantes structurelles peuvent être mobilisées. Il s'agit de mieux caractériser des trajectoires à partir de la compréhension des mécanismes en jeu et des conditions aux limites. Les travaux sur les pièges à pauvreté et les possibilités d'en sortir progressent dans ce sens (Enfors, 2013 ; Lade et al., 2017).

\section{Des blocages résilients}

Le deuxième paradoxe tient au fait que la résilience est recherchée dans le cadre d'interventions d'aide au développement et humanitaire (Janin et Roy, 2016) alors même que des situations de blocage et de pauvreté persistante peuvent être qualifiées de résilientes, du fait de la persistance du sous-développement et de populations marginales, crise après crise.

Les opérateurs de l'aide humanitaire tels que Oxfam ou la Croix-Rouge se réfèrent à la résilience en anticipant que des populations déjà vulnérables vont devoir continuer à faire face à des aléas répétés (Croix-Rouge, 2013 ; Jennings et Manlutac, 2015). Dans les cas les plus difficiles, la résilience relève de la survie, de la possibilité de se reconstruire après un désastre.

\footnotetext{
3 Traduction des auteurs.

${ }^{4}$ Désigne le renforcement du pouvoir d'action et du droit à la parole des femmes.
} 
Cependant, la résilience n'est pas seulement le retour à un état ou une trajectoire initiale, car cet état ou cette trajectoire n'est pas toujours désirable ou fait débat parmi ces opérateurs et autres parties prenantes des interventions. Considérons par exemple, dans un contexte centraméricain, les systèmes agroforestiers (SAF) qui se maintiennent en dépit des cyclones, de mauvaises conditions de marché et de maladies telles que la rouille (Morris et al., 2016). Ces systèmes peuvent être perçus comme résilients du seul fait qu'ils ne disparaissent pas, alors que les rendements sont incertains et les revenus, insuffisants, maintiennent les producteurs dans une situation de pauvreté, et que les jeunes générations cherchent à émigrer. «De nombreuses formes d'agriculture ne sont pas durables bien que résilientes ${ }^{5}$ », résument Hashemi et al. (2017, p. 2) dans le cas de l'agriculture en Iran.

La pérennité de situations de blocage relève de la très grande difficulté à en sortir (Vonthron et al., 2016). L'amélioration ponctuelle des moyens d'existence, avec par exemple la distribution de capital productif, physique ou financier par un projet de développement, pour une nouvelle «activité génératrice de revenus", ne donne pas les effets attendus dans la durée lorsque des rétroactions (Enfors, 2013) empêchent de s'engager dans une dynamique vertueuse de développement (Barrett et Constas, 2014). Ces rétroactions, dans le domaine de l'agriculture et la sécurité alimentaire, sont dues, par exemple : à l'épuisement de ressources naturelles nécessaires à cette nouvelle activité (Lade et al., 2017); à des contraintes exacerbées par le projet et subies par les individus et les ménages les plus vulnérables (manque de temps des femmes en charge des soins aux enfants - Droy et al., 2014); à ce que les ménages préfèrent orienter leurs nouveaux revenus vers des activités qui génèrent de nouvelles contraintes plutôt que de maintenir et accumuler le capital productif censé générer une dynamique vertueuse (Carter et Lybbert, 2012) ; à une réduction de la part d'aliments autoconsommés, à bonne densité nutritionnelle, à la suite de leur commercialisation et à des termes de l'échange défavorables qui réduisent les possibilités de s'approvisionner sur les marchés (Hoddinott et al., 2015, dans le cas du lait en Éthiopie). Les seuils à franchir, sur le plan économique ou en termes de renforcement de capacités par exemple, sont ceux audelà desquels les améliorations structurelles et durables sont possibles. Penser la résilience conduit parfois à envisager la transformation totale de l'ensemble du système et à chercher quels changements le libéreraient des rétroactions bloquantes (Bousquet et al., 2016; Tschakert et Shaffer, 2014). En analysant les mécanismes de ces «pièges à pauvreté ", on peut fournir un éclairage utile, notamment pour le ciblage des ménages vulnérables dans le cadre d'un projet de sécurisation alimentaire. Penser les catégories de bénéficiaires en fonction de la nature des seuils à franchir est une piste pour améliorer le ciblage des projets et dépasser la méthode HEA (Household Economy Approach), dont la pertinence est mise en question (Janin et Roy, 2016 ; Nfon-Dibié et Lankouandé, 2017). Concernant les seuils toujours, la pensée sur la résilience considère aussi, et à l'inverse, les limites à ne pas franchir et au-delà desquelles des changements non souhaitables seraient inéluctables, seuil de non-renouvelabilité des ressources naturelles en particulier. De fait, l'apparente stabilité de nombreuses situations de pauvreté cache le plus souvent des

\footnotetext{
5 Traduction des auteurs.
} 
dynamiques d'affaiblissement et d'augmentation de la vulnérabilité des systèmes (Sanfo et al., 2014 ; Tapsoba et al., 2018). Elles correspondent à des situations non résilientes, qui apparaîtront de façon dramatique en cas de crise, comme cela l'a été lors de la famine au Niger en 2005. En effet, alors même que la production agricole avait baissé d'une façon qui aurait été considérée comme relativement «acceptable » dans le passé, les ménages, qui avaient décapitalisé leurs avoirs, n’ont pas été en mesure d'absorber ce nouveau choc et se sont retrouvés en situation de détresse (Michiels et al., 2012).

Enfin, confondre résilience et blocage est aussi une question de point de vue et d'appréciation, selon les alternatives que l'on considère ou pas, et selon la perception que l'on a des alternatives considérées. Dans l'exemple déjà évoqué de la caféiculture menée avec des pratiques d'agroforesterie peu productive, si le maintien des caféiers a lieu dans une situation où les jeunes sont partis vivre et travailler dans un autre pays d'où ils envoient de quoi subsister à leurs parents, ce maintien peut faire partie d'une stratégie de résilience, illustrant la capacité adaptative de la famille dans son ensemble et les souhaits des jeunes actifs ainsi que ceux de leurs parents attachés à leurs terres. D’un autre point de vue, cette même situation peut être considérée bloquée à de faibles niveaux de productivité, du fait d'un manque de main-d'œuvre et d’investissements pour améliorer les performances de la caféiculture.

Ces exemples soulignent la nécessité de préciser des conditions de ce que serait une «bonne » résilience, de définir des seuils à franchir (Robert et Lallau, 2016). Cela pose la question de ce qu'est un état « désirable », de quel point de vue et comment se fait le passage d'états « non désirables » à « désirables ». Il s'agit de préciser et répondre à ces interrogations avec les populations concernées (Tschakert et Dietrich, 2010) et les contributions de plusieurs disciplines. Ces regards complémentaires sont nécessaires pour distinguer les seuils que l'on veut franchir et les limites à ne pas dépasser (Burkett et al., 2014 ; Barrett et Constas, 2014 ; Botta et Bousquet, 2017).

\section{Une métaphore controversée pour l'action}

Abordons maintenant un troisième paradoxe, lié au précédent : il concerne la dimension - et la volonté mobilisatrice pour l'action de la résilience (Béné, 2017), alors même qu'elle prête à controverses. La résilience est couramment utilisée comme une métaphore, en lien avec les notions de durabilité (Carpenter et al., 2001) et d'adaptation. «La résilience a été consacrée comme une stratégie politique essentielle dans le champ des risques ", écrit Quenault (2015). En matière d'adaptation au changement climatique, c'est à l'aune de la résilience climatique que doivent être décrits les résultats attendus des propositions au Fonds vert pour le climat ${ }^{6}$. Pour le Sahel et l'Afrique de l'Ouest, les « initiatives de résilience » font l'objet d'une «alliance globale» nommée AGIR (Alliance globale pour les initiatives de résilience), une plateforme multisectorielle réunissant les acteurs du développement.

${ }^{6}$ Voir www.greenclimate.fund (consulté en avril 2019). 
Dans ce contexte d'adoption assez unanime du terme, Biggs et al., (2012) et le programme GRAID (Guidance for Resilience in the Anthropocene: Investments for Development) ${ }^{7}$ explicitent sept principes d'action collective et individuelle face à la perturbation et au changement :

1) «entretenir la diversité et la redondance », par exemple la diversité des cultures, des sources de revenus, de l'alimentation, la redondance pour l'approvisionnement ou pour les débouchés des agriculteurs. La redondance correspond à des solutions de rechange en cas de défaillance ou disparition d'une ressource (mauvaise récolte) ou d'un débouché (prix de vente tombé très bas);

2) «gérer la connectivité » entre les niveaux écologiques et sociaux, c'est-à-dire prêter attention à l'importance et la force des liens entre les différentes composantes d'un système, de manière à faciliter la transmission de changements aux conséquences bénéfiques et empêcher ou contenir la transmission de perturbations et de leurs effets néfastes. Dans cette optique, on considérera par exemple le rôle des liens familiaux dans la gestion des crises. Ces liens peuvent à la fois être source d'entraide (partage des repas) et cause de vocations sacrifiées (travail des enfants, mariages précoces);

3) "gérer les variables lentes et les rétroactions », surveiller par exemple l'érosion des sols ou l'effet sur la santé des carences en micronutriments, et pour cela, saisir les évolutions des conditions dans lesquelles sont décidés la production agricole, l’approvisionnement alimentaire et les repas dans le ménage ;

4) «favoriser la réflexion portant sur les systèmes adaptatifs complexes », de façon par exemple à jouer des $\underline{\underline{s y n e r g i e s}}$ et des complémentarités à différents niveaux (entre plantes, entre personnes, entre institutions, etc.) et à considérer les changements et les réorganisations possibles (agencement des cultures et des arbres, répartition des tâches dans un ménage agricole, arrangements sociaux pour l'accès au foncier, etc.) ;

5) «encourager l'apprentissage » et l'expérimentation afin de mieux évaluer les alternatives en présence, tirer les enseignements des événements passés, actualiser ses connaissances compte tenu des évolutions observables et envisageables, tester de nouvelles réponses envisageables à de nouvelles perturbations plus ou moins prévisibles ;

6) «élargir la participation» de façon à tenir compte des différentes logiques d'action à l'œuvre dans un même système. Une attention particulière à la diversité des points de vue en présence, tous légitimes a priori, participe d'un climat de confiance favorable à la robustesse des processus de prise de décision. Il s'agit notamment de considérer collectivement un ensemble de critères décisifs dans les choix de culture ou d'alimentation et dans la poursuite des objectifs de sécurité alimentaire.

7) "promouvoir les systèmes de gouvernance polycentrique ", en favorisant la multiplicité et la coordination des centres de décision et d'action. Par exemple concernant le stockage des céréales et pour

\footnotetext{
${ }^{7}$ Voir http://applyingresilience.org/fr/start-fr//(consulté en avril 2019).s
} 
maintenir des conditions favorables à la sécurité alimentaire, promouvoir les actions coordonnées des opérateurs privés, États, groupements d'agriculteurs, ménages...

Les principes exposés ci-avant traduisent la pensée sur la résilience en termes de propriétés du système socio-écologique et d'attributs clés de sa gouvernance (Biggs et al., 2012). Divers cas sont décrits pour illustrer ces principes et les concepts (Anderies et al., 2006) et, plus largement, chercher à distinguer ce qui peut être considéré comme de la « résilience» et ce qui ne l'est pas (Barrett et Constas, 2014). Dans les faits, la distinction est plus incertaine et relève de l'interprétation des processus en cours. Celle-ci dépend de la façon dont on pose les problèmes de développement et d'insécurité alimentaire, avec quels acteurs, en fonction de quel horizon temporel, à quelle échelle.

Par exemple, concernant le premier principe, la diversité des cultures dans les exploitations familiales est considérée par certains spécialistes comme désirable, bénéfique pour améliorer la diversité alimentaire au sein des exploitations (Jones et al., 2014), source de stabilité des revenus et de préservation, à long terme, des ressources génétiques (Kahane et al., 2013). Mais il est aussi démontré que la diversité des cultures a un effet négatif sur la diversité alimentaire (Sibathu et Qaim, 2018). La controverse tient à la prise en compte de plusieurs dimensions de la sécurité alimentaire, dans un cas, avec une approche systémique, alors que, dans l'autre cas, l'analyse de corrélations est unidimensionnelle.

La marge d'interprétation des principes de résilience peut être vaste, du fait des différences d'approches mais aussi de désaccords sur les modalités des interventions de sécurisation alimentaire, d'incertitudes sur l'accès aux ressources ou encore, des difficultés à tenir compte des populations marginales (Tschakert et Shaffer, 2014). La mobilisation du terme de « résilience» ne permet pas forcément de trancher entre des visions différentes de ce qui est souhaitable (Smith et Stirling, 2010). De plus, traiter de résilience dans le domaine du développement expose aux controverses sur le développement (Rist, 2007) ou sur le besoin d'aide au développement (Moyo, 2009), ou encore sur ce que « s'adapter » veut dire (Fisichelli et al., 2016).

Chercher à résoudre le paradoxe du consensus sur la résilience et des controverses que nourrit son interprétation passe par la participation des populations concernées, leur implication dans l'approche analytique et dans la construction des indicateurs de résilience en fonction de cette analyse.

\section{Intégrer et décomposer}

Un quatrième paradoxe de la résilience concerne sa dimension intégratrice (Béné et al., 2016 ; Folke, 2016 ; Lallau et al., 2018), alors même que les méthodes de mesure de la résilience des ménages consistent essentiellement à distinguer et à jauger différentes catégories d'actifs et capacités mobilisables face aux adversités (Schipper et Langston, 2015).

La dimension intégrative de la résilience explique probablement le succès du terme, déjà évoqué, dans un contexte de changement global et de multiples perturbations. L'apport attendu de la résilience tient notamment à ce qu'elle intègre : 
- différentes échelles de temps, allant du temps court de l'urgence humanitaire au moyen et long termes du développement, qui se traduisent en langage propre au « bailleur »;

- différentes façons de faire face aux chocs : en les absorbant, s'adaptant, en se transformant.

Cette compréhension provient directement de la résilience des SES, qui intègre également :

- différents niveaux d'organisation, spatiaux ou sociaux, imbriqués le plus souvent et aux contours contextuels et évolutifs : les ménages comme ensemble de personnes partageant des ressources et des repas ; les exploitations agricoles, collectifs organisés autour de la production, comprenant des familles des individus et des parcelles qu'ils cultivent; des parcours d'éleveurs, des terroirs villageois, des pays, etc. ;

- différents processus en interaction : écologiques, économiques, sociaux, nutritionnels... ;

- l'expression de différents points de vue, grâce à la participation des populations et des autres acteurs mobilisés ou influençant leur sécurité alimentaire.

Pourtant, lorsqu'il s'agit de mesurer la résilience, certains commencent par distinguer différentes composantes de la résilience. Les méthodes RIMA et COBRA en particulier s'inspirent du cadre d'analyse des moyens d'existence (Scoones, 1998), comme indiqué plus haut. Le guide BRACED rassemble également divers éléments explicatifs de la résilience, avec une boîte à outils de diagnostic, avant de passer à l'élaboration d'un plan d'action. La façon dont plusieurs éléments d'appréciation sont rassemblés, dans un indice de la résilience ou au cœur d'un plan d'action, reste néanmoins peu explicite. Elle relève de calculs statistiques sur les composantes structurelles de la résilience dans RIMA ou du cadre logique d'un projet de développement dans BRACED. Or, si cette intégration est caractéristique de la pensée en termes de « résilience », elle mériterait pour le moins une explicitation et une mise à disposition, via une langue appropriée, pour les différentes parties prenantes.

Résoudre cette ambiguïté sur le caractère intégrateur de la résilience passe, comme pour le premier paradoxe, par la compréhension des mécanismes de la résilience (Gitz et Meybeck, 2012 ; Tittonell, 2014). Pour ce faire, la démarche d'évaluation de la résilience de l'Alliance pour la résilience (Resilience Alliance, 2010) a développé des outils d'analyse des trajectoires d'un SES en fonction des liens entre événements concernant ce SES à différentes échelles et entre phénomènes de différentes natures. Le «profil historique » invite à penser les humains dans leur écosystème à propos d'une problématique (de sécurité alimentaire, par exemple), à situer des événements plus ou moins soudains (une loi sur l'usage des ressources naturelles, un nouveau marché) sur des dynamiques plus longues (choix d'usage des sols, répartition des tâches) et à identifier alors les trajectoires qui résultent de l'interaction de plusieurs processus qui ont des temporalités différentes (Eychène, Fuentes et al., 2013).

Il en résulte une vision dynamique pour penser conjointement différents changements et pour appréhender les questions des impacts de perturbations sur des trajectoires : quelles transmissions des chocs climatiques ou de prix ? Quels enchainements entre des phénomènes de différentes natures et à 
différentes échelles? Comment casser la transmission des effets néfastes d'une catastrophe pour en atténuer les dommages? Ou alors, comment le système (exploitation agricole, territoire...) peut-il se réorganiser et changer de trajectoire selon un scénario plus favorable? Les réponses à ces questions gagnent à être multidisciplinaires - puisqu'il est question de phénomènes de différentes natures - et multipoints de vue, du fait de la dimension interprétative de l'évaluation de la résilience. D'où les enjeux de dialogue entre disciplines et de participation des acteurs concernés pour clarifier les liens entre événements qui ont marqué l'évolution de leur exploitation agricole ou de leur territoire et pour caractériser des trajectoires souhaitables. La démarche est interprétative. Appréhender ainsi la complexité, sans s'y perdre, suppose de s'en tenir aux éléments en interaction, après les avoir définis en fonction du SES considéré à partir d'un problème. Il n'est pas proposé d'outil générique, mais une démarche de conceptualisation en fonction du problème posé.

\section{Ajuster les interventions d'aide ou piloter le système}

L'avant dernier paradoxe se situe dans la relation nouée avec la vulnérabilité. En mettant l'accent sur les capacités de personnes ou de systèmes à faire face à des perturbations, la résilience se démarque d'une vision attentiste de la vulnérabilité (Lallau, 2014). Pourtant, c'est pour rendre compte des interventions d'aide et d'urgence et les réajuster, que la mesure de la résilience se développe le plus depuis une dizaine d'années dans le monde de l'aide internationale.

Résilience et vulnérabilité ont beaucoup été comparées en termes de concepts ou d'approches. Fondamentalement, la principale différence entre les deux cadres conceptuels repose sur les modalités d'analyse de la trajectoire du système et d'articulation avec l'adaptation (Bousquet et al., 2015 ; Buchheit et al., 2016). Les travaux sur la résilience débutent par une analyse du système, de ses composantes comme de leurs interactions, pour comprendre l'histoire et la trajectoire potentielle de ce système. La position de ce dernier sur la trajectoire informe alors des réponses possibles aux perturbations. D’un autre côté, les travaux sur la vulnérabilité débutent par l'analyse des relations entre une perturbation donnée et un objet particulier, et essaient d'évaluer la réponse de cet objet. Ils permettent de définir les états possibles de l’objet résultant de la relation perturbation-réponse, mais ils n’analysent guère les conséquences sur les autres objets, ni en termes de trajectoire générale du système auquel appartient cet objet. La vulnérabilité apparaît généralement comme l'équivalent de ce que l'on appelle la résilience spécifique d'un objet (en d'autres termes la résilience d'un objet à une perturbation donnée, dont on sait qu'elle va faire franchir un seuil). Par exemple, la résilience spécifique d’une famille d'éleveurs à une sécheresse: si celle-ci dure plusieurs mois, le troupeau disparaitra. La résilience générale du système considère tout type de chocs : par exemple comment les pasteurs peuvent survivre dans leur identité d'éleveurs face à des chocs répétés (sécheresse, épidémies) et des changements (urbanisation, sédentarisation). Cette résilience générale correspond au «développement socialement durable» que Ballet et ses co-auteurs (Ballet, Dubois et Mahieu, 2005) appellent de leurs vœux, car elle fait la part belle aux interactions sociales des acteurs, à 
leurs capacités d'agir en introduisant de l'aléa et de la contingence. Elle permet d'expliquer l'innovation, les sauts qualitatifs, les bifurcations de trajectoire.

Si l'on se place dans une perspective gestionnaire, la résilience est mobilisée dans la définition des actions destinées à piloter l'évolution du système vers un état désiré, alors que la vulnérabilité est plutôt employée dans le cadre de réflexions portant sur des actions de gestion visant principalement à réduire les réponses négatives aux perturbations. Dans la pratique, l'évaluation de la vulnérabilité sert à identifier les populations les plus vulnérables et à hiérarchiser les priorités d'interventions pour leur venir en aide. En ce sens l'analyse de la vulnérabilité s'inscrit dans la continuité des analyses de la pauvreté où l'hétérogénéité des situations incite à affiner toujours plus l'analyse.

On pourrait s'attendre à ce que l'évaluation de la résilience, en revanche, serve directement aux acteurs et qu'ils soient beaucoup plus proactifs, contribuant par leurs capacités (Ballet et al., 2013) à l'évolution du système dont ils font partie. Pourtant, la mesure de la résilience se développe essentiellement dans le cadre de l'aide internationale. Conçues pour être favorables aux populations dites "bénéficiaires», les interventions d'aide internationale, de développement ou d'urgence, gagnent à être considérées aussi en termes de perturbations sur un système qui évolue, afin d'analyser ce qu'elles déclenchent.

\section{De la pluridisciplinarité à l'économie standard ?}

Enfin, il est paradoxal qu'un concept pluridisciplinaire (Quinlan et al., 2016) aboutisse, en fin de compte, à des approches classiques en matière de gestion des risques comme en économie du développement autour d'indicateurs quantitatifs (Lallau et al., 2018). En matière de gestion des risques, la résilience se caractérise par des stratégies de minimisation des risques et de recherche de statu quo (Tapsoba et al., 2018). En économie de l'environnement et du développement, la résilience est associée à la durabilité du développement dans un contexte de multiples aléas (Perrings, 2006). Le développement des indicateurs de résilience des ménages s’appuie sur des enquêtes fines de panel (Béné, 2017 ; FAO, 2016). Ces enquêtes examinent en détail les activités, l'accès aux services et aux infrastructures, la nature des actifs, les filets de sécurité et les réseaux sociaux. Les données de panel servent par exemple (Anderson et al. 2017) à calculer des indicateurs de sécurité alimentaire avant et après un choc.

Le caractère dynamique de l'analyse est recherché avec l'augmentation de la fréquence du panel puisque la même population est enquêtée de façon répétée. Le souci de représentativité statistique conduit à envisager des dispositifs lourds où les ménages sont sollicités pour donner un grand nombre d'informations factuelles, et souvent d'ordre "privé », davantage que pour leur point de vue. L'approche participe à l'amélioration d'une sorte de tableau de bord, toujours plus détaillé - et coûteux -, dans le diagnostic et l'aide à la décision au plus haut niveau, qui, eux, sont inaccessibles à ceux dont on évalue la résilience. Elle s’inscrit dans la continuité des grandes enquêtes nationales répétées auprès des ménages, coordonnées par la Banque mondiale, pour estimer, toujours plus précisément, la pauvreté. 
Le « penser résilience » apporte un éclairage différent pour analyser les non-linéarités du développement. Il s'agit pour commencer d'intégrer plusieurs points de vue, de croiser les éléments de compréhension fournis par les acteurs et par diverses disciplines à différentes échelles. Par exemple : les conséquences économiques locales de la hausse internationale du coût des intrants et de la baisse nationale des prix de vente des productions agricoles, les effets des migrations sur les choix de cultures et les pratiques agricoles dans les exploitations ou les impacts écologiques des changements d'usage des sols aux échelles de la parcelle et du territoire. Pour analyser les dynamiques, chaque discipline s'intéresse en particulier aux optimum et aux équilibres. En analyse économique, par exemple, nombre de variables sont exogènes et les effets de retour à l'équilibre sont bien décrits, toutes choses étant égales par ailleurs, ainsi que les possibilités théoriques d'optimiser l'usage des ressources. Lorsque, comme proposé par le guide pratique Resilience Assessment (Resilience Alliance, 2010), plusieurs disciplines se concertent et réfléchissent aux enchainements possibles des dynamiques qu'elles étudient, elles permettent de comprendre que les mécanismes d'équilibrage ne peuvent opérer, d'où un éclairage sur les irréversibilités qu'il est impossible d'obtenir au sein d'une approche unidisciplinaire (Eychène, Fallot et al., 2013). Cet éclairage permet de formuler de nouvelles hypothèses sur les trajectoires favorables à la sécurité alimentaire en fonction de plusieurs dynamiques en interaction. Il reste à les préciser quantitativement et à les valider scientifiquement. L'analyse qualitative préalable aura alors permis de cibler l'analyse quantitative sur quelques aspects cruciaux dans les réponses aux perturbations.

\section{Conclusion : une compréhension transdisciplinaire des trajectoires et des seuils}

Nous avons cherché à préciser ce qui semble spécifique à l'approche «résilience » et utile pour comprendre comment développement et évaluation de résilience peuvent se renforcer mutuellement en pratique.

Six paradoxes de la résilience ont été examinés. Au-delà d'un usage normatif entre experts, l'examen de chaque paradoxe pour une étude donnée concourt à réinterroger les processus de développement en contexte incertain et apporte de nouveaux éclairages sur les stratégies d'intervention. On a cherché comment les résoudre et mieux saisir les liens production agricole et sécurité alimentaire des ménages ruraux dans des contextes de pauvreté et de multiples aléas. En résumé, traiter de résilience consiste à croiser les regards de plusieurs disciplines et des acteurs concernés par des changements ou des perturbations à différentes échelles pour analyser les dynamiques en jeu et leurs interactions. Il s'agit de considérer, à la fois, les «actifs» (assets) et les mécanismes de la résilience, de préciser et de qualifier les limites à ne pas franchir, de caractériser les trajectoires passées et leurs enseignements en termes d'alternatives futures, compte tenu des conséquences possibles d'interventions pour le développement et d'autres perturbations. Ces paradoxes se recouvrent partiellement. Ils relèvent, au final, de deux enjeux de recherche pour caractériser les trajectoires de développement et impliquer réellement les populations concernées. 


\section{$\underline{\text { La caractérisation des trajectoires }}$}

Évaluer la résilience suppose d'analyser comment différents processus s'enchainent ou se neutralisent, amplifiant ou bloquant la transmission d'un événement adverse, facilitant ou empêchant les impacts positifs d'une intervention. Ce type d'analyse en matière de sécurité alimentaire des ménages ruraux repose à la fois sur une connaissance empirique des ménages et sur un travail d'interprétation des liens entre les divers événements et les processus observés. Cette double dimension factuelle et interprétative est essentielle à l'analyse de la résilience. Elle est d'autant plus riche et pertinente qu'elle associe les éléments de compréhension et les visions des acteurs concernés, comme des chercheurs ayant étudié leurs modes de production, d'approvisionnement et de consommation alimentaires.

\section{La coproduction de normes}

La plupart des auteurs et des manuels recommandent de dépasser une utilisation métaphorique, abstraite et désincarnée du concept de résilience, en particulier pour aborder les problèmes de développement et de sécurité alimentaire. Pour cela, il conviendrait de mieux contextualiser les réflexions en insistant: la résilience : de qui, à quoi ? À ces deux questions se rajoute celle de savoir qui définit la résilience, comment la mesurer et quelles sont les interventions pour l'améliorer. L'analyse que nous avons effectuée et l'approche qui en découle s'inspirent des principes de cogestion adaptative (Armitage et al., 2010) ou d'accompagnement (Étienne, 2010). Elle consiste à (i) reconnaître la multiplicité d'acteurs et donc de points de vue sur le développement, l'environnement et la sécurité alimentaire, (ii) favoriser l'interaction entre ces différents points de vue pour collectivement définir quelles sont les situations satisfaisantes et leurs métriques, (iii) identifier et sélectionner des actions, interventions pour conduire la trajectoire vers ces situations désirables.

Les auteurs

\section{Abigail Fallot}

Abigail Fallot est chercheure sur la durabilité des réponses aux changements climatiques, notamment sur l'adaptation des populations rurales et les mesures d'atténuation en agriculture. Elle adopte et développe, en Afrique et en Amérique latine, des approches interdisciplinaires et participatives à l'échelle locale, pour une compréhension partagée des trajectoires de développement envisageables et l'évaluation de stratégies d'adaptation.

A récmment publié

Locatelli B., Aldunce P., Fallot A. et al., 2017, «Research on Climate Change Policies and Rural Development in Latin America: Scope and Gaps », Sustainability, vol. 9, n 10, p.1831. DOI : $\underline{10.3390 / \mathrm{su} 9101831}$ 
François Bousquet

Francois Bousquet a travaillé sur les questions de gestion des ressources renouvelables, de modélisation multi-agents, d'approches d'accompagnement, de résilience des système sociaux et écologiques, des formes d'attachement aux lieux. Il est membre du comité éditorial de Ecology and Society et de Natures, sciences et sociétés, ainsi que de la Resilience Alliance, et organisateur de la conférence de ce réseau en 2014.

A récemment publié

Quinn T., Bousquet F., Guerbois C. et al., 2019, «How Local Water and Waterbody Meanings Shape Flood Risk Perception and Risk Management Preferences », Sustainability Science, n 14, p. 1-14. DOI : 10.1007/s11625-019-00665-0

Brown K., Adger W. N., Devine-Wright P., Anderies J. M., Barr S., Bousquet F. et al., 2019, «Empathy, Place and Identity Interactions for Sustainability», Global Environmental Change, n 56, p. 11-17. DOI : 10.1016/i.gloenvcha.2019.03.003

Bousquet F., Mathevet R., 2019, «Cultural Resilience as the Resilience of a Distinctness. Distinctness from What? for What? », dans Rampp B., Endreß M., Naumann M. (dir.), Resilience in Social, Cultural and Political Spheres, Wiesbaden, Springer, p. 305-321. DOI : 10.1007/978-3-658-15329-8 15

Therville C., Brady U., Barreteau O., Bousquet F. et al., 2018, «Challenges for Local Adaptation when Governance Scales Overlap. Evidence from Languedoc, France», Regional Environmental Change, n 13, p. 1-13. DOI : $\underline{10.1007 / \mathrm{s} 10113-018-1427-2}$

Mathevet R., Bousquet F., Raymond C. M., 2018, « The Concept of Stewardship in Sustainability Science and Conservation Biology », Biological Conservation, $n^{\circ}$ 217, p. 363-370. DOI : $\underline{\text { 10.1016/j.biocon.2017.10.015 }}$

Sandrine Dury

Sandrine Dury, agro-économiste de formation, travaille depuis vingt ans sur la consommation alimentaire, notamment en Afrique. Ses travaux récents portent sur la sécurité alimentaire et nutritionnelle. Elle travaille en particulier sur les liens entre production et alimentation chez les ménages agricoles et sur la diversité des comportements de consommation.

A récemment publié

Ambagna J. J., Dury S., Dop M.C., 2019, «Estimating Trends in Prevalence of Undernourishment: Advantages of Using HCES over the FAO Approach in a Case Study from Cameroon” Food Security, vol. 11, nº 1, p. 93-107. DOI : $10.1007 /$ s12571-018-00884-w

Dury S., Essomba J.-M., 2018, «Afrique centrale», dans Poulain J.-P. (dir.), Dictionnaire des cultures alimentaires, Paris, PUF, p. 15-21, http://agritrop.cirad.fr/591355/ (consulté en avril 2019). 
Ky H., Dury S., 2018, « Productivité agricole et diversité alimentaire au Burkina Faso », Revue Cedres-Études, $\mathrm{n}^{\circ} 65$,

p. $160-177$,

https://agritrop.cirad.fr/587906/1/Ky $\% 20 \mathrm{Habi} \% 26$ Sandrine $\% 20$ DURY $\% 20$ tiré $\% 20 \mathrm{a} \% 20$ part $\% 202$.pdf (consulté en avril 2019).

Gaillard C., Martin S., Bosc P.-M., El-Ati J., Dop M. C., Trabelsi T., Amiot Carlin M.-J., Dury S., 2018, «Explorer les liens entre agriculture et sécurité alimentaire: une enquête auprès des femmes du gouvernorat de Sidi-Bouzid, en Tunisie», Cabiers Agricultures, vol 27, n 1, p. 15501. DOI : $10.1051 /$ cagri/2018005

Dury S., Vall É., Imbernon J., 2017, « Production agricole et sécurité alimentaire en Afrique de l'Ouest », Cabiers Agricultures, vol 26, nº 6, p. 61001. DOI : $10.1051 /$ cagri/2017047 


\section{Bibliographie}

Adger W. N., 2000, «Social and Ecological Resilience: Are They Related? », Progress in Human Geography, vol. 24, n 3, p. 347-364. DOI : 10.1191\%2F030913200701540465

Adger W. N., Hughes T. P., Folke C. et al., 2005, «Social-Ecological Resilience to Coastal Disasters », Science, vol. 309, nº 5737, p. 1036-1039. DOI : $10.1126 /$ science.1112122

Ancey V., Pesche D., Daviron B., 2014, «La résilience, maillon entre urgence et développement ou buzzword palliatif à la crise de la pensée?», Séminaire Sécurité alimentaire, Montpellier, 28 février, https://docplayer.fr/85457667-La-resilience-maillon-entre-urgence-et-developpement-ou-buzzwordpalliatif-a-la-crise-de-la-pensee.html (consulté en avril 2019).

Anderies J., Walker B., Kinzig A., 2006, «Fifteen Weddings and a Funeral: Case Studies and ResilienceBased Management", Ecology and Society, vol. 11, $\mathrm{n}^{\circ}$ 1, art. 21, https://www.ecologyandsociety.org/vol11/iss1/art21/ (consulté en avril 2019).

Code de champ modifié

Anderson C. L., Reynolds T., Merfeld J. D. et al., 2017, «Relating Seasonal Hunger and Prevention and Coping Strategies: A Panel Analysis of Malawian Farm Households », The Journal of Development Studies,

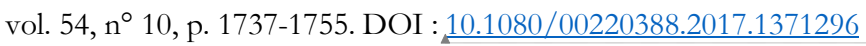

Armitage D., Berkes F., Doubleday N., 2010, Adaptive Co-Management: Collaboration, Learning, and Multi-Level Governance, Vancouver, UBC Press.

Ballet J., Bazin D., Dubois J.-L. et al., 2013, Freedom, Responsibility and Economics of the Person, Londres, Routledge.

Ballet J., Dubois J.-L., Mahieu F.-R., 2005, L'autre développement: le développement socialement soutenable, L'Harmattan, Paris.

Barrett C. B., Constas M. A., 2014, «Toward a Theory of Resilience for International Development Applications ", Proceedings of the National Academy of Sciences, vol. 111, n 40, p. 14625-14630. DOI : $\underline{10.1073 / \text { pnas. } 1320880111}$

Béné C., 2017, «Conceptualizing and Measuring Resilience in the Context of Food Security - Lessons from Low Income Countries", Séminaire sur la sécurité alimentaire, Montpellier, Cirad, 19 juin, https://www.dailymotion.com/video/x5tthkp (consulté en avril 2019).

Béné C., Godfrey Wood R., Newsham A. et al., 2012, «Resilience: New Utopia or New Tyranny? Reflection about the Potentials and Limits of the Concept of Resilience in Relation to Vulnerability Reduction Programmes », IDS Working Paper, n 405. DOI : 10.1111/i.2040-0209.2012.00405.x

Béné C., Headey D., Haddad L. et al., 2016, «Is Resilience a Useful Concept in the Context of Food Security and Nutrition Programmes? Some Conceptual and Practical Considerations », Food Security, vol. 8, $\mathrm{n}^{\circ} 1$, p. 123-138. DOI : $\underline{10.1007 / \mathrm{s} 12571-015-0526-\mathrm{x}}$

Code de champ modifié

Code de champ modifié

Code de champ modifié

Code de champ modifié 
Béné C., Newsham A. Davies M. et al., 2014, «Review Article: Resilience, Poverty and Development »,

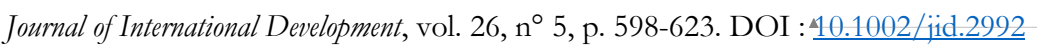

Code de champ modifié

Berkes F., Folke C. (dir.), 1998, Linking Social and Ecological Systems: Management Practices and Social Mechanisms for Building Resilience, Cambridge, Cambridge University Press.

Biggs R., Schlüter M., Biggs D. et al., 2012, «Toward Principles for Enhancing the Resilience of Ecosystem Services", Annual Review of Environment and Resources, vol. 37, n 1, p. 421-448. DOI : $\underline{10.1146 / \text { annurev-environ-051211-123836 }}$

Botta A. Bousquet F., 2017, «La résilience des systèmes écologiques et sociaux : accompagner la prise en compte de l'incertitude pour le développement», Perspective, n ${ }^{\circ} 43$ p. 1-4, http://publications.cirad.fr/une notice.php? dk=584566 (consulté en avril 2019).

Bousquet F., Botta A., Alinovi L. et al., 2016, «Resilience and Development: Mobilizing for Transformation », Ecology and Society, vol. 21, n³ 3. p. 40. DOI : $10.5751 /$ ES-08754-210340

Bousquet F., Robbins P., Peloquin C. et al., 2015, «The PISA Grammar Decodes Diverse HumanEnvironment Approaches», Global Environmental Change, vol.34, p.159-171, http://publications.cirad.fr/une notice.php?dk=576731 (consulté en avril 2019).

BRACED Myanmar Alliance, 2016, «Community Resilience Assessment and Action Handbook», Londres, BRACED, http://www.braced.org/contentAsset/raw-data/127f0e24-a44a-4468-abca96db853f6558/attachmentFile (consulté en avril 2019).

Brand F., Jax K., 2007, « Focusing the Meaning (s) of Resilience: Resilience as a Descriptive Concept and a Boundary Object», Ecology and Society, vol. 12, $\mathrm{n}^{\circ}$ 1, art. 23, http://www.ecologyandsociety.org/vol12/iss1/art23/ (consulté en avril 2019).

Buchheit P., D’Aquino P., Ducourtieux O., 2016, « Cadres théoriques mobilisant les concepts de résilience et de vulnérabilité », Vertigo, vol. 16, nº 1 . DOI : $\underline{10.4000 / \text { vertigo. } 17131}$

Burkett V. R., Suarez A. G., Marco B. et al., 2014, «1. Point of Departure », dans Field C. B. et al. (dir.), Climate Change 2014: Impacts, Adaptation, and Vulnerability. Part A: Global and Sectoral Aspects. Contribution of Working Group II to the Fifth Assessment Report of the Intergovernmental Panel on Climate Change, Cambridge, Cambridge University Press, p. 169-194, https://www.ipcc.ch/site/assets/uploads/2018/02/WGIIAR5Chap1 FINAL.pdf (consulté en avril 2019).

Cannon T., Müller-Mahn D., 2010, «Vulnerability, Resilience and Development Discourses in Context of Climate Change », Natural Hazards, vol. 55, n³, p. 621-635. DOI : 10.1007/s11069-010-9499-4

Carpenter S., Walker B., Anderies J. M. et al., 2001, «From Metaphor to Measurement: Resilience of What to What? », Ecosystems, vol. 4, nº 8, p. 765-781. DOI : 10.1007/s10021-001-0045-9

Code de champ modifié

Code de champ modifié

Code de champ modifié

Code de champ modifié

Code de champ modifié 
Carter M. R., Lybbert T. J., 2012, «Consumption Versus Asset Smoothing: Testing the Implications of Poverty Trap Theory in Burkina Faso », Journal of Development Economics, vol. 99, n 2, p. 255-264. DOI : 10.1016/j.jdeveco.2012.02.003

Croix-Rouge, 2013, «La résilience et la sécurité alimentaire. Un enjeu partagé Nord-Sud », Baromètre de la faim,

2013,

https://www.croixrouge.fr/content/download/759211/14396952/version/1/file/\%20BAROMETRE+DE+LA+FAIM+2 013.PDF (consulté en avril 2019).

Droy I., Pascual C., Bidou J.-É., 2014, «Chapitre 3. Inégalités de genre et vulnérabilité alimentaire au Bénin », dans Guétat-Bernard H., Saussey M. (dir.), Genre et savoirs, Bondy, IRD Éditions, p. 85-115, https://books.openedition.org/irdeditions/9143?lang=fr (consulté en avril 2019).

Dubois J.-L., Ouattara M., 2014, «Vous avez dit "résilience”? Éléments conceptuels et politiques publiques », dans Châtaignier J.-M. (dir.), Fragilités et résilience : les nowvelles frontières de la mondialisation, Karthala, Paris, p. 35-41.

Dubois M., 2016, Table ronde sur la résilience XXXIIe Journées du développement ATM 2016, Lille, 1-3 juin.

Enfors E., 2013, «Social-Ecological Traps and Transformations in Dryland Agro-Ecosystems: Using Water System Innovations to Change the Trajectory of Development», Global Environmental Change, vol. 23, n 1, p. 51-60. DOI : 10.1016/i.gloenvcha.2012.10.007

Étienne M. (dir.), 2010, La modélisation d'accompagnement: une démarche participative en appui au développement durable, Versailles, Éditions Quae.

Eychène C., Fuentes M., Fallot A., 2013, « Construcción participativa del perfil histórico de un territorio », 7th Wallace Conference on Climate-Smart Territories in the Tropics: Production, Mitigation and Adaptation for Improved Well-Being, $\quad$ Montpellier/Cirad-Green,

Turrialba/CATIE-CCC, https://agritrop.cirad.fr/572626/1/document 572626.pdf (consulté en avril 2019).

Eychène C., Fallot A., Brenes C. et al., 2013, «Cascadas de umbrales en un sistema agroforestal con café frente a disturbios del cambio global», Montpellier/Cirad-Green, Turrialba/CATIE-CCC, https://hal.archives-ouvertes.fr/hal-01298736/document (consulté en avril 2019).

FAO, 2015, «SHARP: Self-Evaluation and Holistic Assessment of Climate Resilience of Farmers and Pastoralists », Rome, FAO, http://www.fao.org/3/a-i4495e.pdf (consulté en avril 2019).

FAO, 2016, «RIMA-II: Resilience Index Measurement and Analysis », Rome, FAO, http://www.fao.org/3/a-i5665e.pdf (consulté en avril 2019).

Fisichelli N. A., Schuurman G. W., Hoffman C. H., 2016, «Is 'Resilience' Maladaptive? Towards an Accurate Lexicon for Climate Change Adaptation », Environmental Management, vol. 57, n 4, p. 753-758. DOI : $\underline{10.1007 / \mathrm{s} 00267-015-0650-6}$ 
Folke C., 2016, «Resilience (Republished)», Ecology and Society, vol. 21, n 4, art. 44. DOI : $\underline{10.5751 / \mathrm{ES}-}$ 09088-210444

Folke C., Carpenter S., Walker B. et al., 2010, «Resilience Thinking: Integrating Resilience, Adaptability and Transformability », Ecology and Society, vol. 15, $\mathrm{n}^{\circ} 4, \quad$ art 20, http://www.ecologyandsociety.org/vol15/iss4/art20/ (consulté en avril 2019).

Gitz V., Meybeck A., 2012, "Risks, Vulnerabilities and Resilience in a Context of Climate Change », dans Meybeck A., Lankoski J., Redfern S. et al. (dir.), Building Resilience for Adaptation to Climate Change in the Agriculture Sector: Proceedings of a Joint FAO/OECD Workshop, Rome, OECD/FAO, 23-24 avril, p. 19-36, http://www.fao.org/3/i3084e/i3084e03.pdf (consulté en avril 2019).

Hashemi S. M., Bagheri A., Marshall N., 2017, «Toward Sustainable Adaptation to Future Climate Change: Insights from Vulnerability and Resilience Approaches Analyzing Agrarian System of Iran »,

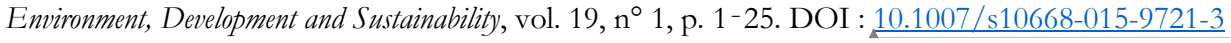

Hoddinott J., Headey D., Dereje M., 2015, «Cows, Missing Milk Markets, and Nutrition in Rural Ethiopia», The Journal of Development Studies, vol.51, nº, p. 958-975. DOI : $10.1080 / 00220388.2015 .1018903$

Holling C. S., 1973, «Resilience and Stability of Ecological Systems », Annual Review of Ecology and Systematics, $\mathrm{n}^{\circ} 4$, p. 1-23. DOI : 10.1146/annurev.es.04.110173.000245.

Janin P., Roy A., 2016, «La «résilience pour la sécurité alimentaire» au Burkina Faso: entre dires, labellisation et (re-)positionnements d'acteurs", Catastrophes, vulnérabilités et résiliences dans les pays en développement, Lille, juin http://hal.ird.fr/ird-01525287/document (consulté en avril 2019).

Jennings S., Manlutac J. I. (dir.), 2015, A Companion Guide to Resilience, Oxfam, https://wwwcdn.oxfam.org/s3fs-public/file attachments/ml-companion-guide-resilience-040216-en 0.pdf (consulté en avril 2019).

Jones A. D., Shrinivas A., Bezner-Kerr R., 2014, «Farm Production Diversity is Associated with Greater Household Dietary Diversity in Malawi: Findings from Nationally Representative Data », Food Policy, $n^{\circ} 46$, p. 1-12. DOI : $10.1016 /$ i.foodpol.2014.02.001

Kahane R., Hodgkin T., Jaenicke H. et al., 2013, «Agrobiodiversity for Food Security, Health and Income», Agronomy for Sustainable Development, vol.33, n 4, p.671-693, http://publications.cirad.fr/ une notice.php?dk=571044 (consulté en avril 2019).

Kevane M., Gray L. C., 1999, «A Woman’s Field Is Made At Night: Gendered Land Rights And Norms In Burkina Faso », Feminist Economics, vol. 5, n 3, p. 1-26. DOI : $\underline{10.1080 / 135457099337789}$

Koffi J. M., 2014, «La résilience : origines et évolutions d'un concept polysémique », dans Châtaigner J. M. (dir.), Fragilités et résilience : les nowvelles frontières de la mondialisation, Paris, Karthala, p. 53-62. 
Korbéogo G., 2016, «Variabilité socio-écologique, crise du pastoralisme et résilience des Peuls pasteurs du Gourma rural (Burkina Faso)», VertigO, vol. 16, n 1. DOI : https://døi.org/10.4000/vertige.17241

Lade S. J., Haider L. J., Engström G. et al., 2017, «Resilience Offers Escape from Trapped Thinking on Poverty Alleviation», Science Advances, vol. 3, $\mathrm{n}^{\circ} 5, \quad$ e1603043. DOI : https://doi.org/10.1126/sciadv.1603043

Lallau B., 2014, « La résilience contre la faim-Nouvelle donne ou nouvel artifice », 8 e Journées de recherches en sciences sociales, Grenoble, Sfer/Inra/Cirad, 11-12 décembre, http://resiliences.univ-lille1.fr/wpcontent/uploads/LALLAU-SFER-décembre-2014.pdf (consulté en avril 2019).

Lallau B., Laissus-Benoist P. Mbetid-Bessane E., 2018, «Introduction : la résilience peut-elle passer de la théorie aux pratiques ?», Revue internationale des études du développement, vol. 3, $\mathrm{n}^{\circ} 235$, p. 9-25. DOI : $\underline{10.3917 / \text { ried.235.0009 }}$

Lourme-Ruiz A., Dury S., Martin-Prével Y., 2016, «Consomme-t-on ce que l'on sème ? Relations entre diversité de la production, revenu agricole et diversité alimentaire au Burkina Faso », Cabiers Agricultures, vol. 25, n 6, p. 65001. DOI : $10.1051 /$ cagri/2016038

LWR (Lutheran World Relief), 2016, The Dynamic Resilience Wheel (DReW), Baltimore, LWR, https://lwr.org/resiliencewheel (consulté en avril 2019).

Mathevet R., Bousquet F., 2014, Résilience et environnement : penser les changements socio-écologiques, Paris, BuchetChastel.

Michiels D., Egg J., Blein R., 2012, «La répétition des crises alimentaires et nutritionnelles au Niger : la rénovation urgente des politiques de sécurité alimentaire », Cabiers Agricultures, vol. 21, n 5, p. 302-310. DOI : 10.1684 /agr.2012.0588

Morris K. S., Méndez V. E., Van Zonnenfeld M., 2016, Agroecology and Climate Change Resilience: In Smallbolder Coffee Agroecosystems of Central America, décembre, Rome, Bioversity International, https://ccafs.cgiar.org/publications/agroecology-and-climate-change-resilience-smallholder-coffeeagroecosystems-central\#.XK97SK3pNE4 (consulté en avril 2019).

Moyo D., 2009, Dead Aid: Why Aid is Not Working and How There Is a Better Way for Africa, Basingstoke, Palgrave Macmillan.

Nfon-Dibié A., Lankouandé E., 2017, «Ciblage HEA et courtage d'un projet de sécurité alimentaire et nutritionnelle dans la Boucle du Mouhoun (Burkina Faso) : quels ajustements et compromis ? ", Colloque international Sécurisation alimentaire: entre savoirs et actions, Nogent sur Marne, IEDES Université Paris 1 Panthéon-Sorbonne, 16 octobre.

O’Connell D., Abel N., Grigg N. et al., 2016, Designing Projects in a Rapidly Changing World: Guidelines for Embedding Resilience, Adaptation and Transformation into Sustainable Development Projects, Washington, Global
Code de champ modifié

Code de champ modifié

Code de champ modifié

Code de champ modifié

Code de champ modifié 
Environment Facility, http://www.stapgef.org/the-resilience-adaptation-and-transformation-assessment-

framework (consulté en avril 2019).

Pelling M., 2011, Adaptation to Climate Change: From Resilience to Transformation, Londres, Routledge.

Perrings C., 2006, «Resilience and Sustainable Development», Environment and Development Economics, vol. 11, n 4, p. 417-427. DOI : $\underline{10.1017 / \text { S1355770X06003020 }}$

Quenault B., 2015, «De Hyōgo à Sendai, la résilience comme impératif d'adaptation aux risques de catastrophe: nouvelle valeur universelle ou gouvernement par la catastrophe?», Développement durable \& Territoires, vol. 6, nº 3. DOI : 10.4000/developpementdurable.11010

Quinlan A. E., Berbés-Blázquez M., Haider L. J. et al., 2016, « Measuring and Assessing Resilience: Broadening Understanding through Multiple Disciplinary Perspectives », dans Allen C. (dir.), Journal of Applied Ecology, vol. 53, n 3, p. 677-687. DOI : $10.1111 / 1365-2664.12550$

Resilience Alliance, 2010, «Assessing Resilience in Social-Ecological Systems: Workbook for Practitioners. Version 2.0.», Handbook of Sustainability Assessment, p. 285, https://www.resalliance.org/resilienceassessment (consulté en avril 2019).

Rist G., 2007, «Development as a Buzzword », Development in Practice, vol. 17, $\mathrm{n}^{\circ}$ 4-5, p. 485-491. DOI : $\underline{10.1080 / 09614520701469328}$

Robert P., Lallau B., 2016, « Mesurer la résilience des ménages ruraux sénégalais: Une approche en termes de trajectoires et seuils de moyens d'existence", Éthique et Économique, vol. 13, n 2, p. 29-47, http://ethique-economique.net/Volume-13-Numero-2.html (consulté en avril 2019).

Sanfo S. Gérard F., Fallot A. et al., 2014, « Resilience of Small Farmers Facing Global Change: The Case of the Ganzourgou Province, Plateau Central, Burkina Faso », Resilience 2014, Montpellier, 4-8 mai.

Sanou K., Badolo M., 2017, « Corpus de référentiels d'aide à la décision pour la résilience de la production vivrière aux risques climatiques dans la région Maritime au Togo", VertigO, vol. 17, $\mathrm{n}^{\circ} 3$. DOI : $\underline{10.4000 / \text { vertigo. } 18859}$

Schipper E. L. F., Langston L., 2015, «A Comparative Overview of Resilience Measurement Frameworks: Analysing Indicators and Approaches", ODI Working Paper, $\mathrm{n}^{\circ}$ 422, juillet, https://www.odi.org/sites/odi.org.uk/files/odi-assets/publications-opinion-files/9754.pdf (consulté en avril 2019).

Scoones I., 1998, «Sustainable Rural Livelihoods. A Framework for Analysis », IDS Working Paper, n 72, janvier, https://www.ids.ac.uk/publications/sustainable-rural-livelihoods-a-framework-for-analysis/ (consulté en avril 2019).

Code de champ modifié

Code de champ modifié

Code de champ modifié 
Sibhatu K. T., Qaim M., 2018, «Farm Production Diversity and Dietary Quality: Linkages and

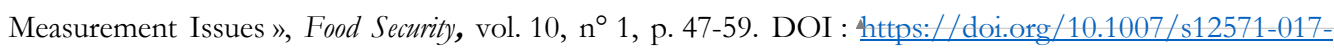
$\underline{0762-3}$

Smith A., Stirling A., 2010, «The Politics of Social-Ecological Resilience and Sustainable Socio-Technical Transitions", Ecology and Society, vol. 15, $\mathrm{n}^{\circ}$ 1, https://www.ecologyandsociety.org/vol15/iss1/art11/ (consulté en avril 2019).

Tapsoba A. Gérard F., Daré W., 2018, «Grands périmètres irrigués et résilience des paysans au Sahel: Le cas de Bagré au Burkina Faso", Revue internationale des études du développement, vol. 3, n 235, p. 147-176. DOI : $\underline{10.3917 / \text { ried } .235 .0147}$

Tittonell P., 2014, «Livelihood Strategies, Resilience and Transformability in African Agroecosystems », Agricultural Systems, $\mathrm{n}^{\circ} 126$, p. 3-14. DOI :

Tschakert P., Dietrich K., 2010, «Anticipatory Learning for Climate Change Adaptation and Resilience », Ecology and Society, vol. 15, n ${ }^{\circ}$, art 11, https://www.ecologyandsociety.org/vol15/iss2/art11/ (consulté en avril 2019).

Tschakert P., Shaffer L. J., 2014, «Ingredients for Social-Ecological Resilience, Poverty Traps, and Adaptive Social Protection in Semi-Arid Africa », dans Sakai S., Umetsu C. (dir.), Social-Ecological Systems in Transition, Tokyo, Springer Japan, p. 139-156.

UNDP (United Nations Development Programme), 2014 , Community Based Resilience Analysis (CoBRA) Conceptual Framework and Methodology, UNDP/DRRAP, https://www.undp.org/content/undp/en/home/librarypage/environmentenergy/sustainable land management/CoBRA/cobra-conceptual-framework.html (consulté en avril 2019).

Vonthron S., Dury S., Fallot A. et al., 2016, «L'intégration des concepts de résilience dans le domaine de la sécurité alimentaire : regards croisés», Cabiers Agricultures, vol. 25, n 6, art. 64001. DOI : $\underline{10.1051 / \text { cagri/2016039 }}$

\section{Code de champ modifié}

Code de champ modifié

Code de champ modifié

Code de champ modifié

Code de champ modifié

Code de champ modifié 
Tableau 1: Principales initiatives en matière d'évaluation de la résilience pour les projets de développement agricole et sécurité alimentaire

* robustesse, auto-organisation, redondance, échelle, rapidité, flexibilité, égalité. ** d'absorption, d'adaptation, de transformation. *** croissance inclusive, sécurité alimentaire, bien-être, qualité de vie et moyens d'existence durables 


\begin{tabular}{|c|c|c|c|c|c|c|}
\hline Méthode ou outil & RIMA & BRACED & COBRA & RAPTA & Manuel RA & Drew \\
\hline Auteur ou porteur & $\begin{array}{l}\text { FAO : } \\
\text { organisation } \\
\text { internationale }\end{array}$ & $\begin{array}{l}\text { DFID : } \quad \text { coopération } \\
\text { britannique }\end{array}$ & $\begin{array}{l}\text { PNUD: organisation } \\
\text { internationale }- \text { "Centre } \\
\text { de politique globale sur } \\
\text { écosystèmes résilients et } \\
\text { désertification» }\end{array}$ & \begin{tabular}{llll} 
STAP du & GEF : & panel \\
d'experts & & \multicolumn{2}{c}{ chercheurs } \\
australiens & du & CSIRO) \\
pour & une & organisation \\
internationale & &
\end{tabular} & $\begin{array}{l}\text { Resilience Alliance : } \\
\text { organisation de recherche }\end{array}$ & $\begin{array}{l}\text { Lutheran World Relief: } \\
\text { organisme caritatif }\end{array}$ \\
\hline Document & $\begin{array}{l}\text { Rapport } \\
\text { décrivant la } \\
\text { méthode de } \\
\text { mesures } \\
\text { directes et } \\
\text { indirectes, } 80 \mathrm{p} \text {. }\end{array}$ & $\begin{array}{l}\text { Manuel pour l'évaluation } \\
\text { et l'action, } 76 \mathrm{p} \text {. }\end{array}$ & $\begin{array}{l}\text { Directives de mise en } \\
\text { œuvre de l'évaluation et de } \\
\text { l'action, 36-24 p. }\end{array}$ & $\begin{array}{l}\text { Directives pour intégrer la } \\
\text { résilience dans les projets, } \\
112 \mathrm{p} .\end{array}$ & $\begin{array}{l}\text { Manuel pour l'évaluation } \\
\text { de résilience dans les } \\
\text { socio-écosystèmes, } 54 \mathrm{p} \text {. }\end{array}$ & $\begin{array}{l}\text { Présentation d'un outil de } \\
\text { caractérisation de la } \\
\text { résilience, } 22 \mathrm{p} \text {. }\end{array}$ \\
\hline Pour qui ? & $\begin{array}{l}\text { Experts } \\
\text { économistes }\end{array}$ & $\begin{array}{ll}\text { Opérateurs } & \mathrm{du} \\
\text { développement } & \end{array}$ & $\begin{array}{l}\text { Équipe multi-agences de } \\
\text { développement } \\
\text { facilitateurs }\end{array}$ & $\begin{array}{l}\text { Concepteurs de projets de } \\
\text { développement durable }\end{array}$ & $\begin{array}{lr}\text { Praticiens } & \text { du } \\
\text { développement } & \text { et } \\
\text { scientifiques } & \end{array}$ & Parties prenantes d'un projet \\
\hline $\begin{array}{l}\text { En quoi consiste } \\
\text { l'évaluation de } \\
\text { résilience? }\end{array}$ & $\begin{array}{l}\text { Analyse } \\
\text { quantitative } \\
\text { économétrique, } \\
\text { mesures } \\
\text { directes et } \\
\text { indirectes (selon } \\
\text { corrélations) } \\
\text { "pour la } \\
\text { conception, }\end{array}$ & 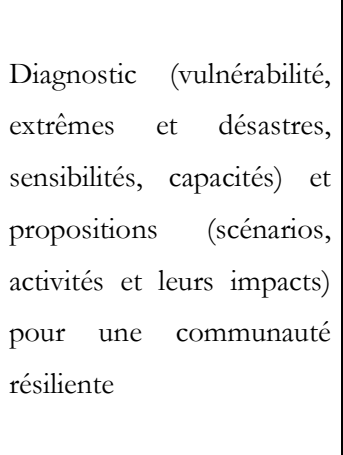 & $\begin{array}{llr}\text { Analyse qualitative : des } \\
\text { moteurs et facteurs } & \text { de } \\
\text { résilience locale ; } & \text { des } \\
\text { stratégies des } & \text { ménages } \\
\text { résilients; } & & \text { des } \\
\text { interventions } & & \text { de } \\
\text { renforcement } & \text { de } & \text { la } \\
\text { résilience } & & \end{array}$ & $\begin{array}{llr}\text { Processus } & \text { itératif d'analyse } \\
\text { systémique } & & \text { des } \\
\text { dynamiques } & \text { (causal } & \text { loops), } \\
\text { approche } & \text { qualitative } & \text { en } \\
\text { termes } & \text { de } & \text { chemins } \\
\text { d'impact, } & & \text { analyse } \\
\text { quantitative } & \text { selon données } \\
\text { disponibles, } & \text { accent } & \text { sur } \\
\text { l'apprentissage } & \end{array}$ & $\begin{array}{l}\text { Analyse transdisciplinaire } \\
\text { des dynamiques et de leurs } \\
\text { interactions sur plusieurs } \\
\text { échelles, approche } \\
\text { qualitative puis quantitative } \\
\text { pour l'identification de } \\
\text { seuils et de changements } \\
\text { en cascade }\end{array}$ & 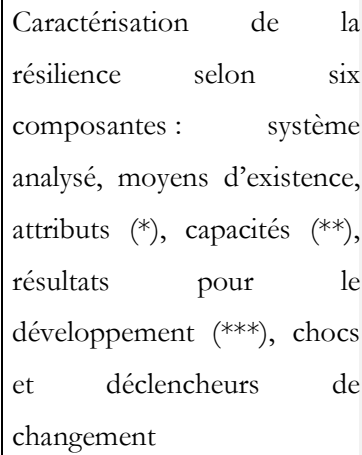 \\
\hline
\end{tabular}




\begin{tabular}{|l|l|l|l|l|l|}
\hline l'exécution, & le \\
suivi & et \\
l'évaluation & de & & & & \\
l'assistance aux & & & & & \\
populations & & & & & \\
démunies » & & & & \\
\hline
\end{tabular}

RIMA (Resilience Index Measurement and Analysis)

BRACED (Building Resilience and Adaptation to Climate Extremes and Disasters)

COBRA (Community Resilience Assessment And Action)

RAPTA (Resilience, Adaptation Pathways and Transformation Assessment)

Manuel RA (Assessing Resilience in Social-Ecological Systems de l'Alliance pour la résilience - Resilience Alliance)

DReW (Dynamic Resilience Wheel)

Sources : FAO, 2016 ; BRACED Myanmar Alliance, 2016 ; UNDP, 2014 ; O’Connell et al., 2016 ; Resilience Alliance, 2010 ; LWR, 2016. 